\title{
Reduced Port Technique for Bariatric Surgery Procedures: Feasibility, Technique and Outcome
}

\author{
Ahmed Elhoofy, MD Mahmoud Zakaria, MD; MRCS \\ Department of General Surgery, Ain Shams University, Cairo, Egypt
}

\begin{abstract}
Aim: Reduced Port laparoscopic Surgery (RPLS) involves fewer ports or narrower ports than standard laparoscopic surgery. It has the potential to cause reduced postoperative pain, reduce abdominal wall trauma and increase the aesthetic result without compromising the outcome. This review describes the feasibility, present situation and challenges faced as well as standardized procedures and the future prospects of reduced port laparoscopic gastrectomy for various bariatric procedure.
\end{abstract}

Materials and methods: From December 2015 to January 2017, 251 patients were enrolled in our trial all of which underwent the procedure using the three port technique. All patients had morbid obesity with history of failure of conservative treatment. The aim was to assess and evaluate postoperative pain and patients' satisfaction with the aesthetic results as well as to define the features of early post-operative complications of patients in comparison to the conventional five ports technique.

Results: 251 patients were enrolled in our trial. The analyzed population included 185 women and 66 men. Mean age and BMI were $41 \pm 23$ and $53 \pm 18$, respectively. Mean operative time was $47 \pm 20$ min for sleeve gastrectomy, $65 \pm 13 \mathrm{~min}$ for mini-gastric bypass and $133 \pm 25 \mathrm{~min}$ for revisional surgeries. Mortality was nil. Overall morbidity rate was recorded. Median duration of hospital stay was 1.5 days (range, 1-2) for sleeve gastrectomy and gastric bypass and 2.5 days (2-4 days) for revisional surgeries.

Conclusion: Three ports technique for bariatric surgery is a safe and effective surgical option in patients with morbid obesity without additional early surgical complications or increase in operative time, and with a greater patient aesthetic satisfaction.

Key word: Reduced port, bariatric surgery.

\section{Introduction}

Reduced port laparoscopic surgery (RPLS) involves fewer ports or narrower ports than standard laparoscopic surgery. ${ }^{1}$ It is a concept that has grown out of the various efforts aimed at minimally invasive surgery. It can be done safely and offer good cosmetic outcomes. $^{2}$

In recent years, reduced port surgery (RPS), has been used in various surgeries, such as splenectomy, colectomy, and gynecologic surgeries, and its cosmetic merit has been widely accepted. ${ }^{3}$ In Western countries, RPS has been used to perform sleeve gastrectomy in bariatric surgery. ${ }^{4}$ It has the potential to cause reduced postoperative pain, reduce abdominal wall trauma and increase the aesthetic result without compromising the outcome.

Our study aimed at establishing the feasibility of usage of three ports instead of the conventional five ports technique in various bariatric procedures; including laparoscopic sleeves gastrectomy, mini-gastric bypass, $R / Y$ gastric bypass and revision of failed bariatric procedure i.e. post vertical band gastroplasty (VBG). This review describes the present situation and challenges faced as well as standardized procedures and the future prospects of reduced port laparoscopic gastrectomy for various bariatric procedure.

\section{Patients and methods Objectives}

The main purpose of our trial was to define the safety of the use of three port technique compared to the classical five port technique in various bariatric surgery procedures.

\section{Ethical and administrative information}

A special informed consent was explained and signed by all patients. All patients accepted the anonymous form of publication for scientific purposes. We declared that there would not be any communication of personal data to third parties, in order to respect patients' privacy.

In this trial, we decided to blind our data analyst in order to avoid any possible error or bias.

\section{Indications, inclusion, and exclusion criteria}

Between December 2015 to January 2017; 251 consecutive patients were enrolled in the trial. Each patient was evaluated by a multidisciplinary team before surgery. The indication for bariatric surgery procedure was following the recommended indications of the 
International Federation for the Surgery of Obesity (IFSO). For the bariatric surgeon, the indications were chronic morbid obesity with a body mass index (BMI) $\geq 40$ or $\geq 35 \mathrm{~kg} / \mathrm{m} 2$ with associated co morbidities, the failure of conservative treatment, and patient age between 16 and 65 years. All patients underwent preoperative abdominal ultrasonography in order to determine the degree of hepatomegaly.

All patients that accepted the research study prospectives and the risks/benefits of having the three port technique surgery were included in the study. Exclusion criteria included patients with a BMI $\geq 75 \mathrm{~kg} /$ $\mathrm{m} 2$, patients younger than 16 years old or older than 65 years old, and all the contraindications to major surgery. In the cases of an undetected large hiatus hernia, we had to change the surgery to a gastric bypass procedure.

For ethical reasons, we included in our protocol that any patients with significant intraoperative bleeding $(>500 \mathrm{~cm} 3$ ) should be converted from the three to five port technique or to open surgery and thus would be excluded from the trial.

\section{Endpoints}

The endpoint was to define the differences in the early post-operative complications of patients who underwent the three port technique versus the traditional five port technique as well as to evaluate any differences between our technique and the five port technique as regard the post-operative pain and patient satisfaction of the esthetic result.

\section{Preoperative Preparations}

Preoperative work up for reduced port technique was the same as for conventional laparoscopic bariatric surgery. This includes evaluation by a multidisciplinary team including nutritionist, endocrinologist, gastroenterologist, cardiologist, pneumologist, psychiatrist, psychologist, anesthesiologist, and bariatric surgeon. Preoperative education was important for good postoperative outcome. It included advice on preoperative low calorie diet intake for at least 2 to 4 weeks, in order to shrink the size of the liver, and evaluate the patient's compliance. Prophylaxis of deep vein thrombosis (DVT) was achieved using both chemical and mechanical modalities (prophylactic dose of low molecular weight heparin (LMWH) 12 hours prior to surgery and compression stockings against thromboembolism). Surgical prophylaxis is achieved by intravenous antibiotic administration just before making the skin incision.

\section{Operative Technique Operative strategy for reduced port laparoscopic sleeve gastrectomy RPLSG}

Our technique in RPLSG included 3-port approach, with no liver retractor. Retraction of fatty liver in the morbidly obese presented unique difficulties during retraction. Fatty liver was retracted by internal retraction by using the mobilized portion of the stomach, complete posterior dissection of the gastric fundus; and anterior eversion of the gastric fundus before the last 2 firings. The surgeon stood between the legs of the patient and the camera assistants stood on the right side of the patient, who was placed in the French (reverse Trendelenburg) position with both upper limbs placed in abduction. A monitor was placed above the patient's head and to the left. Pneumoperitoneum was created with $14 \mathrm{mmHg}$ using a Verress Needle (Ethicon Endo-Surgery Inc., Johnson \& Johnson, Cincinnati, OH, USA) in the left upper quadrant. Once pneumoperitoneum was established, a $12 \mathrm{~mm}$ port for the camera was placed at a distance about 15 to $20 \mathrm{~cm}$ below the xiphoid process 3 to $5 \mathrm{~cm}$ lateral to the midline on the left side. Two additional ports were placed: a $15 \mathrm{~mm}$ port for the surgeon's left hand in the right midclavicular line, $2 \mathrm{~cm}$ above the horizontal line crossing the optical port for the stapler and a $5 \mathrm{~mm}$ port for the surgeon's right hand at the location of the Veress needle in the left upper quadrant (Figure 1A). The greater curvature of the stomach was freed, starting from the middle of the stomach, with a bipolar vessel sealer and divider (LigaSure Atlas ${ }^{\circledR}$; Covidien Ltd., Norwalk, CT, USA) that was introduced through the 5-mm left lateral port. The approach of the lesser sac at this level was easier. With atraumatic forceps that were introduced through the $15 \mathrm{~mm}$ port, the posterior surface of the stomach was raised anteriorly and, caudally, the liver was retracted without direct contact. The mobilization extended from a point 4 to $6 \mathrm{~cm}$ proximal to the pylorus and extended all the way up the greater curvature to the angle of His, staying close to the wall of the stomach, dividing both gastrocolic and gastrosplenic ligaments then mobilization of the angle of His to expose the left crus of the diaphragm. This gentle facilitated complete resection of the fundus. Gentle medial and anterior retraction of the free portion of the greater curvature allowed for adequate exposure of the short gastric vessels without using liver retractor. LigaSure was used to take down retrogastric adhesions. This allowed complete stomach mobilization, excluded the fundus from the gastric sleeve, and removed any redundant posterior wall of the sleeve (Figure 2). Once the stomach was freely dissected, a $36 \mathrm{~F}$ rigid calibration tube is inserted orally by the anesthesiologist and directed through the pylorus and placed against the lesser curvature. This calibrated the size of the gastric sleeve, prevented constriction at the gastroesophageal junction and incisura angularis, and provided a uniform shape to the entire stomach. Any significant small hiatal hernia was reduced, with additional simple, crural closure when indicated. The stomach was then transected starting 4 to 6 $\mathrm{cm}$ proximal to the pylorus. Gastric resection was performed using a gastrointestinal flexible endostapler (EndoGIA Roticulator $\AA$; Covidien Ltd., Norwalk, CT, USA). The stapler was then fired consecutively along the length of the orogastric tube until the angle of His is reached. During the last two firing of the stapler, it was necessary to have a satisfactory posterior visual control of the staple line in order to avoid injury to the spleen. This was easily achieved with the cooperation 
of the stapler traction and the inclination of the camera so that the sleeved stomach should not be narrow at the incisura angularis (Figure 3 ). The stomach was inspected anteriorly as well as posteriorly to be sure that no redundant posterior stomach iwasleft behind. Approximately $80 \%$ of the stomach was separated during this procedure. No reinforcement of the suture line was carried out unless needed. The staple line was checked for any bleeding which was controlled using endoclips. Methylene blue leak test was done to check the integrity of the staple line. The resected stomach was extracted through the $15 \mathrm{~mm}$ port site without endobag. Closure of port sites muscular defect was done by proline 1 suture to avoid post operative port sites hernias. Closure of port sites is done by $3 / 0$ absorbable subcuticular suture with drain insertion through the $5 \mathrm{~mm}$ port.

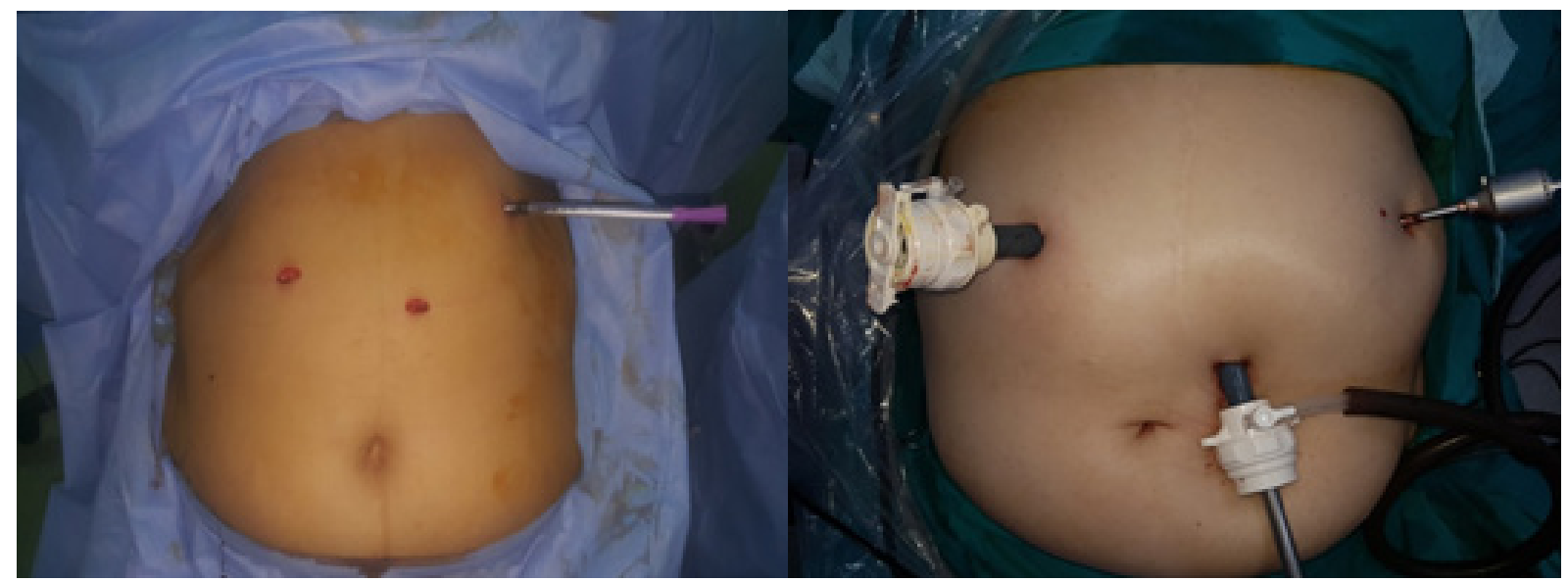

Fig 1: A- Port site placement in RPLSG, B- Port site placement in revision of previous failed open vertical band gastroplasty.
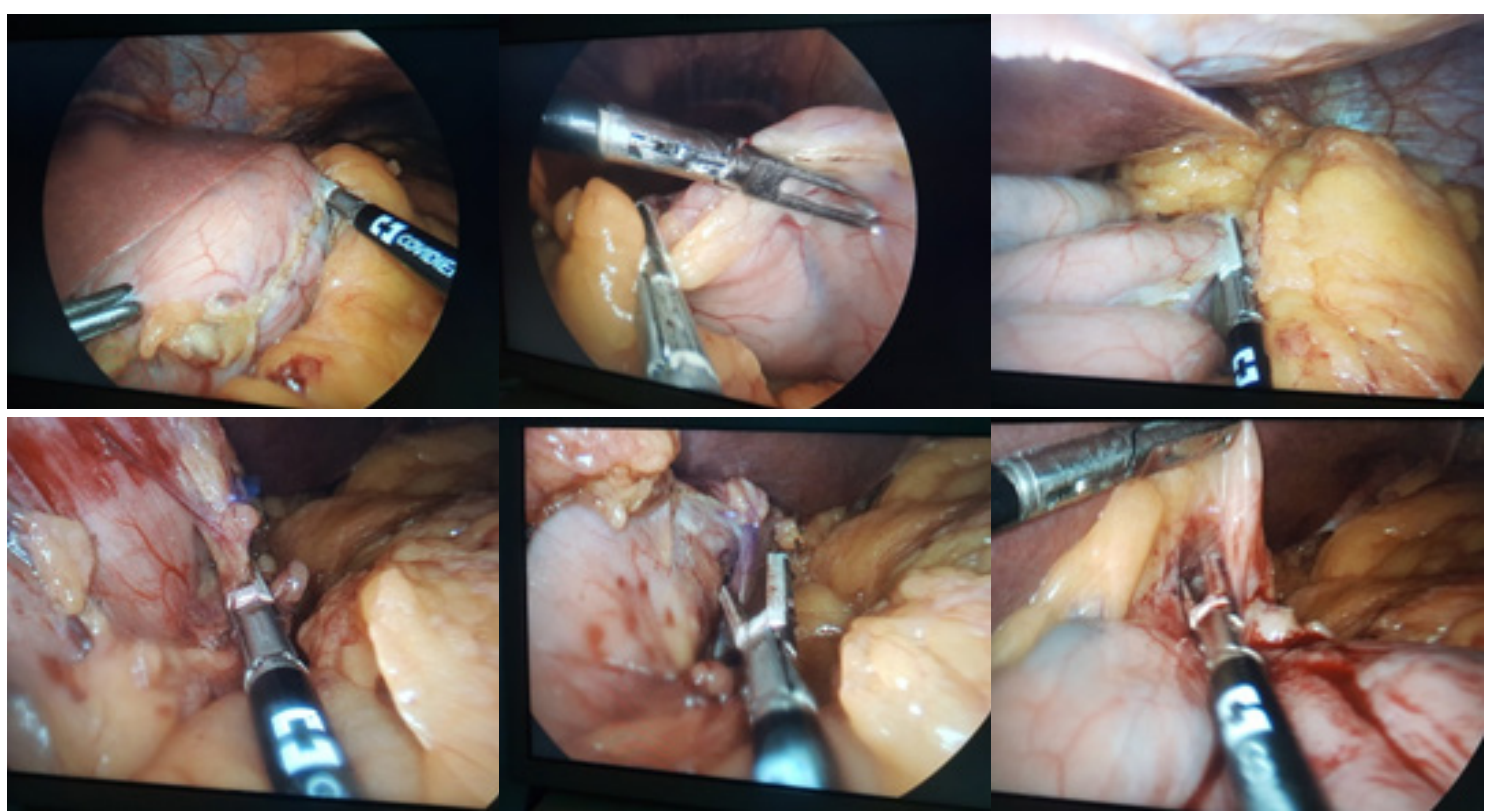

Fig 2: Mobilization and dissection of the stomach from the greater omentum up to the gastroesophageal junction. The standard fourth port for the liver retractor is replaced by the left hand of the surgeon. The surgeon uses the posterior part of the stomach to lift up the left lobe of the liver. The dissection of the stomach is performed completely posteriorly with no anterior dissection. After complete dissection of the attachments of the posterior part of the fundus, the left crus is identified and represents the main landmark of the dissection. From posterior to anterior, a tunnel is created at the level of angle of His. During dissection, the last short gastric vessels are not divided, playing the role of the assistant exposure. The non divided short gastric vessels replace the fifth port from the standard technique. 


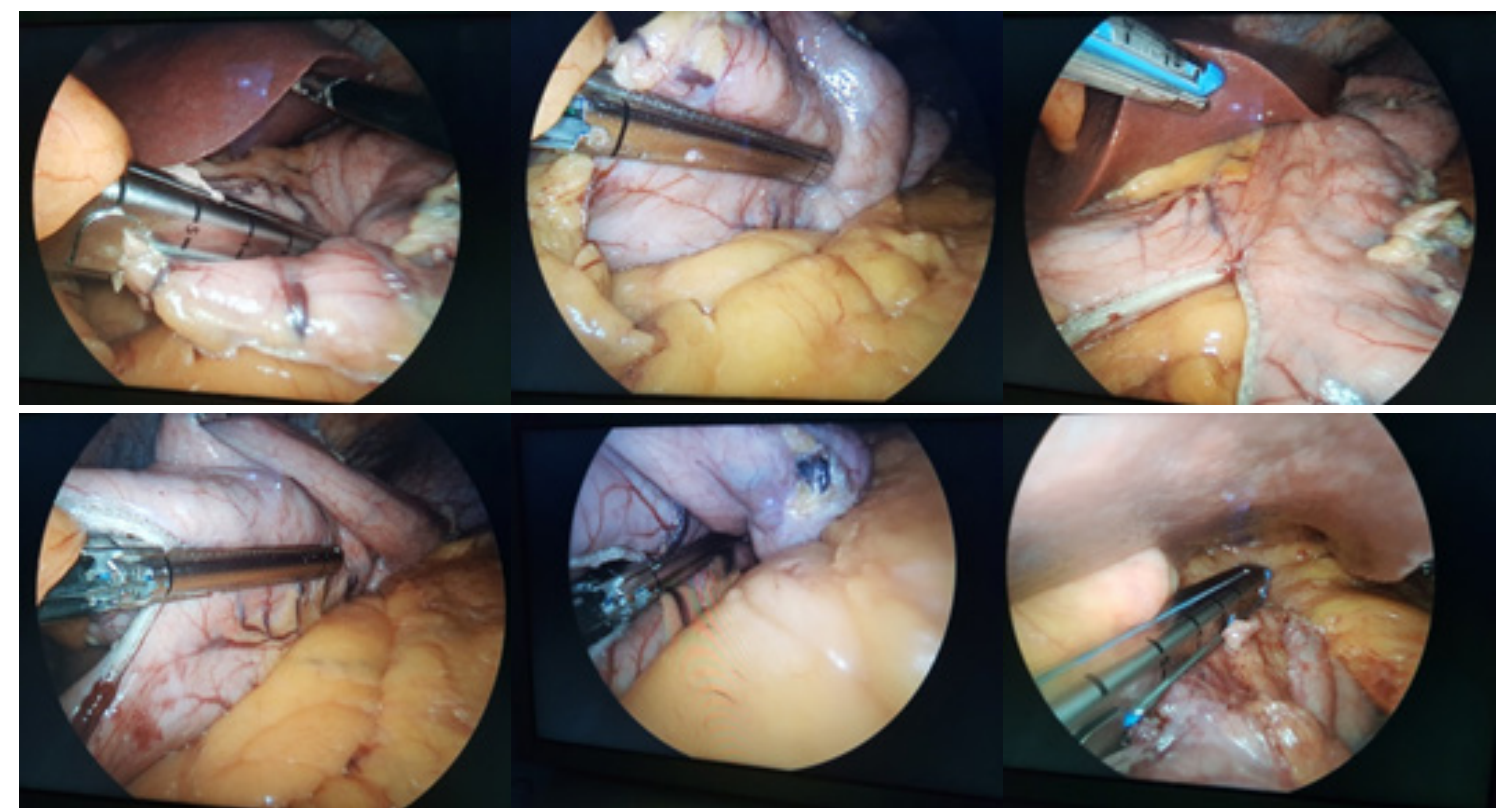

Fig 3: Gastric resection. During the last two firing of the stapler, it is necessary to have a satisfactory posterior visual control of the staple line in order to avoid injury to the spleen. This is easily achieved with the cooperation of the stapler traction and the inclination of the camera. The sleeved stomach should not be narrow at the incisura angularis.

Operative technique for reduced port laparoscopic other bariatric surgery procedures A similar approach was utilized for reduced port laparoscopic mini-gastric bypass RPLMGBP, R/Y gastric bypass and revision of failed bariatric procedure. The surgical technique of reduced port laparoscopic mini-gastric bypass (RPLMGBP) involved placement of 3 ports as before (Figures 1B). A window was created to enter the lesser sac using LigaSure through which the stomach was divided at the junction of the body and the antrum at the level of the crow's foot with 45-mm Endo-GIA stapler to get the longest possible gastric pouch. The standard fourth port for the liver retractor was replaced by the left hand of the surgeon. In addition; in revisional bariatric procedures (ie. Post VBG) the privilege of the presence of the frequently noticed massive adhesion between the diaphragm and the liver minimized the need of the conventional fourth port for liver retraction. This minimized the unexpected injuries to the liver that may be elicited by the liver retraction or prolonged compression of the left lobe ie. PMVT. The surgeon used the posterior part of the stomach to lift up the left lobe of the liver. The dissection of the stomach was then performed completely posteriorly with no anterior dissection. A lesser curvature-based tube of stomach was constructed with a 60-mm linear stapler using 3.5$\mathrm{mm}$ blue cartridges (EndoGIARoticulator $\AA$; Covidien Ltd., Norwalk, CT, USA) around an orogastric tube of 36 Fr size. A jejunal loop, $200 \mathrm{~cm}$ distal to the ligament of Treitz, was then brought up antecolic and anastomosed to the stomach tube with 45-mm Endo-GIA stapler. The common stapling defect was closed over the nasogastric tube with one layer of No 2-0 absorbable V-LocTM suture (Autosuture Division of Covidien, USA) in a running fashion then reinforced by seromuscular 2-0 absorbable Vicryl continuous suture (Figure 4). The anastomosis was then tested with methylene blue injected through the nasogastric tube.

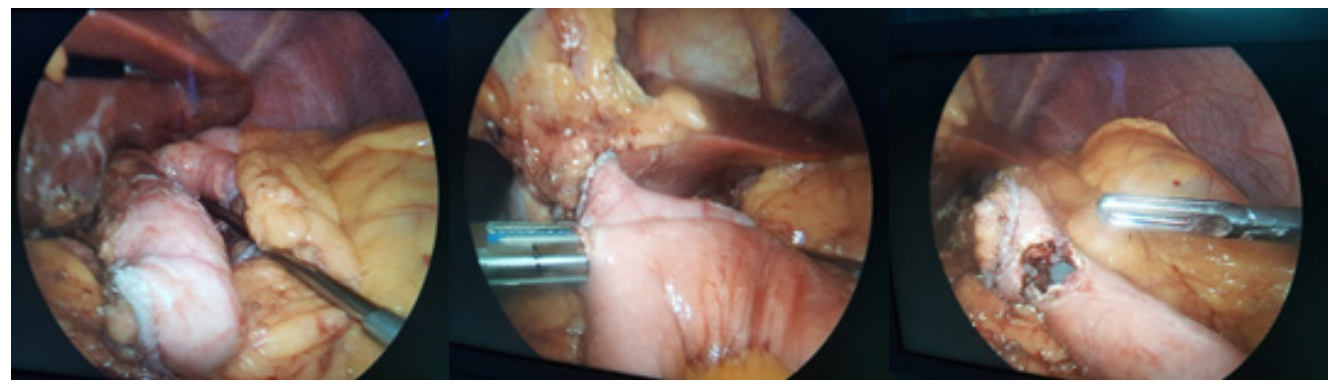

Fig 4: Creation of the gastric pouch and gastrojejunal anastomosis using reduced port technique. 


\section{Postoperative Care}

Patients received postoperative fluids to maintain adequate urine output. Early ambulation was required. Patients used to start clear liquid diet 24 hours post operative. Patients were discharged by the first postoperative day taking adequate oral fluids ( $>2 \mathrm{~L} / \mathrm{d})$. Treatment with the low-molecular-weight heparin (LMWH) enoxaparin (Clexane $R$; Sanofi-Aventis, Paris, France) at $40 \mathrm{mg}$ per day was started 12 hours preoperatively and continued daily for 14 days. Patients started walking within 4-6 hours of surgery with assistance. Postoperative gastrografin swallow was not done routinely. The patient was discharged once she or he was hemodynamically stable, afebrile, ambulating, tolerating a bariatric full liquid diet, and pain could be managed with oral analgesics.

\section{Postoperative follow up and assessment}

The aim was to assess and evaluate postoperative pain and patients' satisfaction with the aesthetic results as well as to define the features of early post-operative complications of patients in comparison to the conventional five ports technique. The short outcomes follow-up included: operative time, conversion, transfusions, fistula, reinterventions and parietal herniation at one and three months after surgery.

\section{Results}

251 patients were enrolled in our trial (173 patients underwent sleeve gastrectomy, 56 underwent minigastric bypass, 3 underwent R/Y gastric bypass, 2 underwent SASI, 11 patients post VBG revision to gastric bypass, 3 patients post sleeve gastrectomy revision to mini-gastric bypass, 2 patients post gastric band revision to mini-gastric bypass and 1 patient post placation revision to $\mathrm{R} / \mathrm{Y}$ gastric bypass). The analyzed population included 185 women and 66 men. Mean age and BMI were $41 \pm 23$ and $53 \pm 18$, respectively. Comorbidities were present in 58 patients $(23.1 \%)$. 51 patients had type 2-diabetes mellitus (20.3\%), 37 patients had hypertension (14.74\%), 33 patients had hyperlipidemia (13.14\%), 23 had obstructive sleep apnea $(9.1 \%)$ and 17 patients had gastroesophogeal reflux disease $(6.7 \%)$. Mean operative time was $47 \pm$ $20 \mathrm{~min}$ for sleeve gastrectomy, $65 \pm 13 \mathrm{~min}$ for minigastric bypass and $133 \pm 25$ min for revisional surgeries. Mortality was nil. Overall morbidity rate was recorded. No inadvertent hollow viscera injury occurred. Transient liver bleeding occurred in 7 patients $(2.8 \%)$ and was managed conservatively during the procedure (i.e., compression, local hemostatic agents). Short gastric bleeding occurred in 3 patients $(1.2 \%)$. No patient required splenectomy. Intraoperative methylene blue test was negative for leakage in all patients $(0 \%)$. One patient $(0.4 \%)$ had postoperative leakage after revision of a case of gastric plication to mini-gastric bypass for which reconversion to $R / Y$ gastric bypass was done laparoscopicaly. No post bariatric stricture, stomal ulcers, portal vein thrombosis, pulmonary embolism or development of fistula were recorded. Median duration of hospital stay was 1.5 days (range, 1-2) for sleeve gastrectomy and gastric bypass and 2.5 days ( $2-4$ days) for revisional surgeries. Two patients $(0.8 \%)$ received blood transfusion post operatively due to postoperative bleeding. No patient developed an incisional hernia to date. Conversion to four ports procedure was necessary in three patients $(1.2 \%)$ and to open surgery in one patient $(0.4 \%)$ (Post VBG patient). Mean excess weight loss (\% EWL) was $17.31 \pm 7.8 \%$ at 1 month, $48.18 \pm 17.1 \%$ at 6 months.

\section{Discussion}

With the recent advances in minimally invasive surgery, there is an increasing interest in surgical techniques that minimize abdominal wall trauma. This facilitated the development of a new concept, reduced port laparoscopy (RPL) with decrease in either the number of ports or the size of ports, or a combination of the two.

In 2008, Saber et al. described the technique of reduced port laparoscopic (RPLS) sleeve gastrectomy (SG). ${ }^{4}$ The approach has been applied to a wide variety of procedures, both bariatric as well as nonbariatric, including appendectomy, cholecystectomy, colectomy, and, more recently, bariatric surgery. The technique is particularly attractive for the placement of an adjustable gastric band, which requires an incision large enough to insert the band and the port, and for the sleeve gastrectomy to allow retrieval of the gastric specimen. ${ }^{5-10}$

To the best of our knowledge, this is one of the first clinical trials that assessed the feasibility of three port technique in laparoscopic mini-gastric bypass and various revisional bariatric surgeries (56 underwent mini-gastric bypass, 3 underwent $R / Y$ gastric bypass, 2 underwent SASI, ${ }^{11}$ patients post VBG revision to gastric bypass, 3 patients post sleeve gastrectomy revision to mini-gastric bypass, 2 patients post gastric band revision to mini-gastric bypass and 1 patient post plication revision to $\mathrm{R} / \mathrm{Y}$ gastric bypass).

The reduced-port approach involves a critical learning curve, thus increasing the technical difficulty of the surgical procedure. The procedure also requires significant coordination between the surgeon and the camera holder.

Vincenzo Consalvo et al. ${ }^{11}$ conducted a study comparing perioperative outcomes of RPLSG versus conventional multi-port LSG. This study found a significant difference in the mean operative time between the two groups. The mean operative time was lesser in the five-trocar technique (43.1 $18.5 \mathrm{~min}$ with a range of 30-66 $\mathrm{min}$ ) compared to the three-trocar technique $(51.5 \pm 10.53$ min with a range of 35-71 $\mathrm{min}$ ), and the statistical analysis showed a two-tailed $P$ value of 0.0004 , which was considered extremely significant. In our study, the mean operative time was $47 \pm 20$ min for sleeve gastrectomy, $65 \pm 13 \mathrm{~min}$ for mini-gastric bypass and $133 \pm 25 \mathrm{~min}$ for revisional surgeries which is comparable to the conventional five port technique in various bariatric surgery procedures. 
Luca Arruet al.; ${ }^{12}$ conducted a study on the threeport laparoscopic sleeve gastrectomy technique; they assessed its feasibility and short outcomes in 25 consecutive super-obese patients. They stated that they observed that fewer incisions (using a 12-mm access incision specifically) later translate into less postoperative discomfort and better esthetic results, as well as a reduction in parietal trauma, pain and risk of hernia. Our study further demonstrated significantly less postoperative pain and need for analgesia as well as greater cosmetic satisfaction at 6 months in the three port technique.

In conclusion, the three-port technique for bariatric surgery could be a comfortable and effective alternative to the standard laparoscopic approach in order to reduce parietal trauma, as well as postoperative pain and with a greater patient aesthetic satisfaction. The three-port technique described herein for patients with morbid obesity or hepatomegaly is safe, technically feasible and reproducible, as shown by the low rate of conversion to a standard laparoscopic procedure (1.2\%) and by the Low rate of conversion to laparotomy $(0.4)$. The interventions in our series were performed by different surgeons and included surgeons-in-training, which demonstrates that it is an easily reproducible technique. This is probably due to the possibility of using conventional instruments and to operate with comfortable instrument triangulation. By reducing the parietal risk, three-port technique improves the results obtained with the standard laparoscopic technique, with no increased risk for patients. Only prospective, randomized clinical trials could determine its effectiveness in terms of evidence-based medicine.

\section{References}

1. Naito T. Chapter 3 Terminology. In: Mori T \& Dapri G (eds). Reduced port laparoscopic surgery. Tokyo: Springer Japan. 2014; 23-26.

2. Noriyuki Inaki: Reduced port laparoscopic gastrectomy: A review, techniques, and perspective. Asian J Endosc Surg. 2015; 8: 1-10.

3. Saber $A A$, Elgamal $M H$, Itawi $E A$ et al: Single incision laparoscopic sleeve gastrectomy (SILS): A novel technique. Obes Surg. 2008; 18: 1338-1342.

4. Hideki Kawamura, Toshiro Tanioka, Kazuaki
Shibuya, Munenori Tahara, Masahiro Takahashi: Comparison of the invasiveness between reducedport laparoscopy-assisted distal gastrectomy and conventional laparoscopy- assisted distal gastrectomy. Int Surg. 2013; 98: 247-253.

5. Saber AA, El-Ghazaly $\mathrm{TH}$, Dewoolkar AV, Slayton SA: Single incision laparoscopic sleeve gastrectomy versus conventional multiport laparoscopic sleeve gastrectomy: Technical considerations and strategic modifications. Surg Obes Relat Dis. 2010; 6: 658-64.

6. Saber AA, El-Ghazaly TH, Elian A, Dewoolkar AV: Single-incision laparoscopic placement of adjustable gastric band versus conventional multiport laparoscopic gastric banding: A comparative study. Am Surg. 2010; 76: 1328-1332.

7. Tacchino RM, Greco F, Matera D, Difl umeri G: Single-incision laparoscopic gastric bypass for morbid obesity. Obes Surg. 2010; 20: 1154-1160.

8. Saber AA, El-Ghazaly TH, Elian A: Single-incision transumbilical laparoscopic sleeve gastrectomy. $\boldsymbol{J}$ Laparoendosc Adv Surg Tech A. 2009; 19: 755-758; discussion 759.

9. Saber AA, El-Ghazaly TH: Early experience with single-access transumbilical adjustable laparoscopic gastric banding. Obes Surg. 2009; 19: 1442-1446.

10. El-Ghazaly TH, Saber AA: Single incision laparoscopic surgery (SILS ${ }^{\mathrm{TM}}$ ) and trocar reduction strategies for bariatric procedures. In: Deitel M, Gagner M, Dixon JB, Himpens J, Madan AK, editors. Handbook of obesity surgery. Toronto: FDCommunications. 2010: 190-197.

11. Consalvo V, Salsano V, Sarno G, Chaze I: Threetrocar sleeve gastrectomy vs standard five-trocar technique: A randomized controlled trial. Obes Surg. 2017; 27: 3142-3148.

12. Arru L, Azagra JS, Goergen M, De Blasi V, De Magistris L, Facy O: Three-port laparoscopic sleeve gastrectomy: Feasibility and short outcomes in 25 consecutives super-obese Patients. Cirugia Espanola (English edition). 2013; 91(S): 279-348. 